\title{
Gapless Surface Dirac Cone in Antiferromagnetic Topological Insulator $\mathrm{MnBi}_{2} \mathrm{Te}_{4}$
}

\author{
Yu-Jie Hao®, ${ }_{1}$ Pengfei Liu, ${ }^{1}$ Yue Feng $\odot,{ }^{1}$ Xiao-Ming Ma, ${ }^{1}$ Eike F. Schwier, ${ }^{2}$ Masashi Arita, ${ }^{2}$ Shiv Kumar $\odot,{ }^{2}$ Chaowei Hu, ${ }^{3}$ \\ Rui'e Lu, ${ }_{1}^{1}$ Meng Zeng, ${ }_{1}^{1}$ Yuan Wang $\odot,{ }^{1}$ Zhanyang Hao $\odot,{ }^{1}$ Hong-Yi Sun, ${ }^{1}$ Ke Zhang, ${ }^{2}$ Jiawei Mei, ${ }^{1}{ }^{1}$ i Ni, ${ }^{3}$

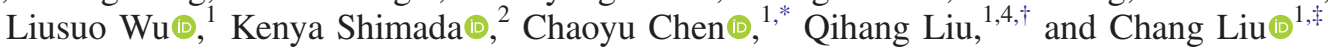 \\ ${ }^{1}$ Shenzhen Institute for Quantum Science and Engineering (SIQSE) and Department of Physics, \\ Southern University of Science and Technology (SUSTech), Shenzhen, Guangdong 518055, China \\ ${ }^{2}$ Hiroshima Synchrotron Radiation Center, Hiroshima University, \\ 2-313 Kagamiyama, Higashi-Hiroshima 739-0046, Japan \\ ${ }^{3}$ Department of Physics and Astronomy and California NanoSystems Institute, \\ University of California, Los Angeles, California 90095, USA \\ ${ }^{4}$ Guangdong Provincial Key Laboratory for Computational Science and Material Design, \\ Southern University of Science and Technology, Shenzhen, Guangdong 518055, China
}

(Received 10 July 2019; revised manuscript received 7 August 2019; published 21 November 2019)

\begin{abstract}
The recently discovered antiferromagnetic topological insulators in the $\mathrm{Mn}$-Bi-Te family with intrinsic magnetic ordering have rapidly drawn broad interest since its cleaved surface state is believed to be gapped, hosting the unprecedented axion states with a half-integer quantum Hall effect. Here, however, we show unambiguously by using high-resolution angle resolved photoemission spectroscopy that a gapless Dirac cone at the (0001) surface of $\mathrm{MnBi}_{2} \mathrm{Te}_{4}$ exists inside the bulk band gap. Such an unexpected surface state remains unchanged across the bulk Néel temperature, and is even robust against severe surface degradation, indicating additional topological protection. Through symmetry analysis and ab initio calculations we consider different types of surface reconstruction of the magnetic moments as possible origins giving rise to such linear dispersion. Our results unveil the experimental topological properties of $\mathrm{MnBi}_{2} \mathrm{Te}_{4}$, revealing that the intrinsic magnetic topological insulator hosts a rich platform to realize various topological phases by tuning the magnetic or structural configurations, and thus push forward the comprehensive understanding of magnetic topological materials.
\end{abstract}

DOI: $10.1103 /$ PhysRevX.9.041038

\author{
Subject Areas: Condensed Matter Physics, \\ Materials Science, \\ Topological Insulators
}

\section{INTRODUCTION}

The integration of magnetic order and topological nontriviality has received much attention since the dawn of the topological era in condensed matter physics [1-4]. In these systems, the absence of time-reversal symmetry $(\mathcal{T})$ brings about a series of exotic quantum phases such as a Chern insulator [5] and axion insulator [6], leading to potential applications in the fields of spintronics and quantum computing. As a renowned example, the quantum anomalous Hall (QAH) effect in Chern insulators promises novel emergent phenomena such as Majorana fermions and

\footnotetext{
*chency@sustech.edu.cn

†liuqh@sustech.edu.cn

Hiuc@sustech.edu.cn
}

Published by the American Physical Society under the terms of the Creative Commons Attribution 4.0 International license. Further distribution of this work must maintain attribution to the author(s) and the published article's title, journal citation, and DOI. anyon statistics [7]. Another distinct topological phase is the axion insulator state, signified by a gapped surface state by magnetization but half-quantized surface Hall conductance, which was proposed to host the topological magnetoelectric effect and axion electrodynamics [6,8]. The QAH insulator was first discovered in a magnetically doped topological insulator (TI) at an ultralow temperature of $30 \mathrm{mK}$ [9]. Proposals of heterostructure engineering based on magnetic insulators and TIs are also expected to realize the QAH effect through magnetic proximity effects, but are challenging in the material choice and interface fabrication [6]. On the other hand, the realization of an axion insulator requires a TI layer sandwiched by two magnetic layers whose moments point to opposite directions [10]. Though the QAH effect and the axion state have been discovered in TIs and magnetic topological heterostructures where ferromagnetically ordered moments are induced by chemical doping [9-13], an intrinsic, stoichiometric magnetic topological insulator (MTI) is highly desired in both cases, as the emerging temperatures of these macroscopic quantum states would otherwise be severely suppressed due to disorder. 

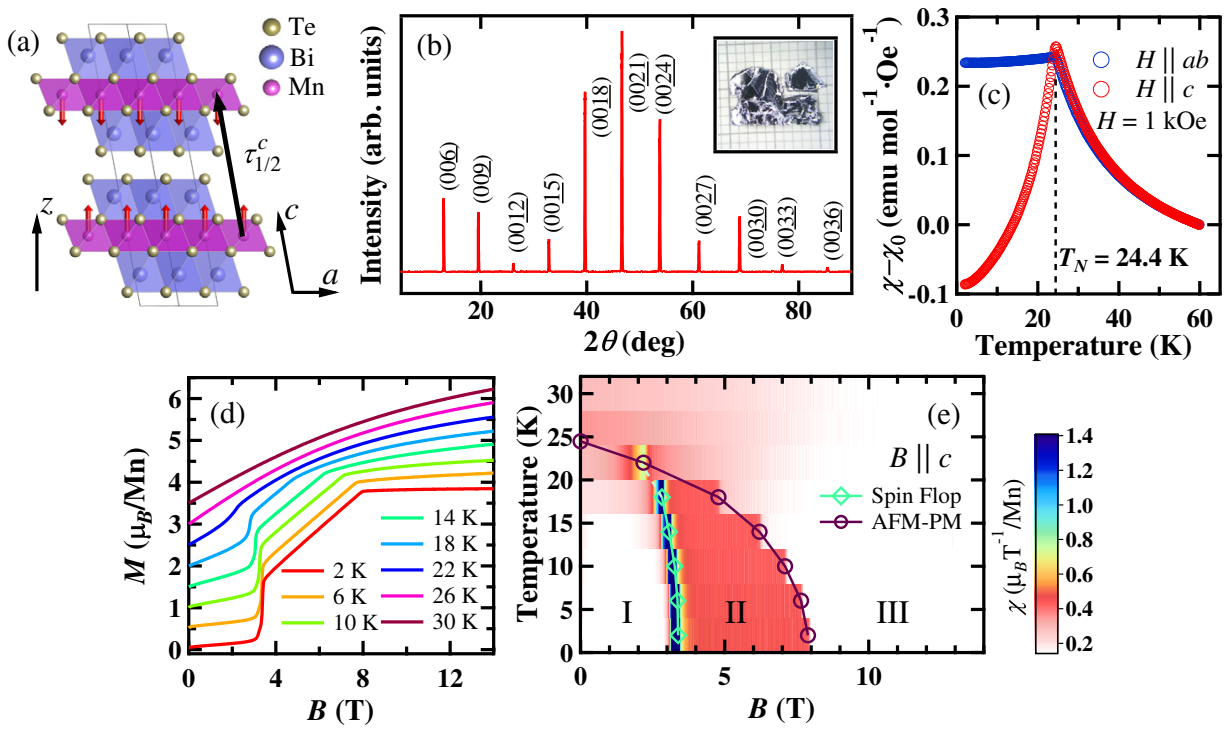

FIG. 1. Crystal characterization and magnetic responses of $\mathrm{MnBi}_{2} \mathrm{Te}_{4}$. (a) Crystal structure and $A$-type AFM magnetic configuration. (b) Single crystal x-ray diffraction data. Inset: Crystal against a millimeter grid. (c) Magnetization curves in two different configurations, $H \| a b$ and $H \| c$. The susceptibility at $60 \mathrm{~K}\left(\chi_{0}\right)$ is subtracted. (d) Field dependence of magnetization $M$, measured at different $T$, with field along the $c$ axis. (e) The field-temperature phase diagram with applied field along the $c$ axis. As $B$ increases, the system evolves from an $A$-type AFM state (I) to a spin-flop AFM state (II), then to a high- $T$ PM state (III).

For both QAH insulators and axion insulators, materials that best fit the above requirement should be magnetic layered compounds having net magnetization and zero magnetization, respectively. Interestingly, both conditions can be achieved in a single material base comprising $A$-type antiferromagnetism (AFM) with out-of-plane magnetic moments, in which the two distinct topological phases can be switched simply by controlling the number of layers. Such a three-dimensional material base is an AFM TI characterized by a novel $\mathbb{Z}_{2}$ topological invariant protected by the product of $\mathcal{T}$ and a half-cell translation along the $c$ axis $\tau_{1 / 2}^{c}$, named $\mathcal{S}^{c}=\mathcal{T} \tau_{1 / 2}^{c}$ [14]. While layered AFM insulators like $\mathrm{CrI}_{3}$ fulfill the magnetic structure but lack a nonzero topological invariant [15], a novel family of van der Waals layered single crystalline materials $\mathrm{MnBi}_{2 n} \mathrm{Te}_{3 n+1} \quad(n=1,2, \ldots) \quad[16,17]$, exemplified by $\mathrm{MnBi}_{2} \mathrm{Te}_{4}$ [18-36], has been established very recently to be stoichiometric TIs with an A-type AFM ground state. The basic magnetic building block of $\mathrm{MnBi}_{2} \mathrm{Te}_{4}$ consists of seven atomic layers that arrange as Te-Bi-Te-Mn-Te-Bi-Te [18-21], called a septuple layer (SL) [Fig. 1(a)]. Its magnetic moments in the bulk are theoretically predicted $[22,23]$, and then confirmed by neutron diffraction experiments [24], to be ferromagnetically (FM) ordered within a Mn plane pointing along the out-of-plane $z$ direction but antiferromagnetically aligned between adjacent Mn layers [Fig. 1(a)]. It was experimentally found that few-SL films of $\mathrm{MnBi}_{2} \mathrm{Te}_{4}$ can realize not only the axion state but a topological transition between the axion state and the QAH state when switching the number of SLs between even and odd numbers $[25,26]$. Furthermore, nine layers of
$\mathrm{MnBi}_{2} \mathrm{Te}_{4}$ is experimentally reported to be a higher-order Chern insulator [27]. Such rich emergent physics render $\mathrm{MnBi}_{2} \mathrm{Te}_{4}$ an ideal platform for studying the interplay between magnetism and topology.

Now that several theoretical predictions and experimental observations point to a simple $A$-type AFM ground state in bulk $\mathrm{MnBi}_{2} \mathrm{Te}_{4}$, there are still substantial discrepancies between the ideal scenario and realistic quantum transport behaviors. For example, while the QAH effect was predicted to appear in an odd number of $\mathrm{MnBi}_{2} \mathrm{Te}_{4}$ layers with uncompensated $A$-type AFM configuration, such an effect was observed experimentally only under a strong magnetic field $(>5 \mathrm{~T}$ ) that forces the AFM ground state to a FM one $[25,26]$. This implies that the inherent magnetic configuration, including the magnitude, orientation, domain, and bulk-surface correspondence, adds complication to the full understanding of intrinsic MTIs such as $\mathrm{MnBi}_{2} \mathrm{Te}_{4}$. Since the key property for the realization of an axion state is the gapped Dirac cone induced by intrinsic magnetization, it is crucial to verify the existence of such a gapped surface state in bulk $\mathrm{MnBi}_{2} \mathrm{Te}_{4}$. In this paper, we profile the topological nature of $\mathrm{MnBi}_{2} \mathrm{Te}_{4}$ by our experimental discovery of the unexpected bulk-surface correspondence using high-resolution angle resolved photoemission spectroscopy (ARPES). Unlike previous theoretical predictions and experimental observations claiming a sizable magnetic gap at the (0001) surface state where $\mathcal{S}^{c}$ is broken [18-20,28], we show unambiguously that there exists an $\mathrm{X}$-shaped, gapless, Dirac cone at this surface, traversing the bulk band gap of $\mathrm{MnBi}_{2} \mathrm{Te}_{4}$. This state is intrinsic to the $\mathrm{MnBi}_{2} \mathrm{Te}_{4}$ crystals, reproducible in all samples we 
measured, free of $k_{z}$ dispersion, unchanged across the bulk magnetic ordering temperature, and is even robust against severe surface degradation. The gapped bands observed by previous works near the Dirac point, on the other hand, are proven to be of bulk nature, having clear $k_{z}$ dispersion. By performing symmetry analysis and density functional theory (DFT) calculations, we attribute the origin of the observed gapless Dirac cone to surface-mediated reconstruction of magnetic moments that differ from the bulk, with the discussion of several proposed occasions including spin disorder, $A$-type AFM with in-plane moment and intralayer AFM. The possibility of surface structural deformation is also discussed. Our work reveals an important factor that can significantly affect the topological property of $\mathrm{MnBi}_{2} \mathrm{Te}_{4}$, i.e., the surface magnetic or structural reconstruction, and thus brings about a more comprehensive understanding of magnetic topological materials in general.

\section{CRYSTAL AND MAGNETIC PROPERTIES OF BULK MnBi $\mathrm{Te}_{4}$}

We begin our discussion by presenting the physical properties measured on the $\mathrm{MnBi}_{2} \mathrm{Te}_{4}$ samples used in our ARPES measurements. It is important to point out that our samples were grown by two different research groups [University of California, Los Angeles (UCLA) and Southern University of Science and Technology (SUSTech)] using slightly different growth procedures, yet the transport, magnetic, and ARPES measurements reveal quantitatively the same results, each on multiple samples, signifying the reliability and repeatability of our data. Figure 1(b) presents the single crystal x-ray diffraction data, as well as the appearance of the crystals, which agree quantitatively with those in the literature. No signal from other crystalline phases is seen. This proves that our photoemission data do not come from impurity phases.

In Figs. 1(c)-1(e) we present the magnetic measurement results, demonstrating that $\mathrm{MnBi}_{2} \mathrm{Te}_{4}$ has an $\mathrm{AFM}$ ground state and a rich magnetic phase diagram. Figure 1(c) shows the magnetization versus temperature curves for two different configurations, $H \| a b$ and $H \| c$. An AFM-paramagnetic (PM) transition is found at $T_{N}=24.4 \mathrm{~K}$, consistent with data from other groups. Figure 1(d) illustrates the isothermal magnetizations of $\mathrm{MnBi}_{2} \mathrm{Te}_{4}$ as a function of applied field along the $c$ axis ranging from 2 to $30 \mathrm{~K}$. All the magnetization curves for $T<T_{N}$ show an abrupt change around the field between 2 and 3.6 T. This suggests the occurrence of a spin-flop-type transition, below which the spin direction of Mn ions turns perpendicular to the easy axis ( $c$ axis). Finally, the magnetization approaches saturation around $M=3.8 \mu_{B} / \mathrm{Mn}$ at $2 \mathrm{~K}$ above $8 \mathrm{~T}$, well consistent with previous results $[24,29,30]$. The fieldtemperature phase diagram of $\mathrm{MnBi}_{2} \mathrm{Te}_{4}$ is depicted in Fig. 1(e). Below $T_{N}$, the critical field of the spin-flop transition $B_{c 1}$ divides the phase diagram into two regions.
At region $\mathrm{I}, \mathrm{MnBi}_{2} \mathrm{Te}_{4}$ shows an $A$-type $\mathrm{AFM}$ order consisting of FM layers coupled antiferromagnetically along the $c$ axis [24]. Above $B_{c 1}$ (region II), it is possible that the moments first turn perpendicular to the $c$ axis due to the lower ground energy, then rotate continuously towards the field direction. When the critical field $B_{c 2}$ is reached, all the moments are polarized along the applied field (region III).

\section{ROBUST SURFACE DIRAC CONE BY ARPES MEASUREMENTS}

We show in Fig. 2 the electronic structure of $\mathrm{MnBi}_{2} \mathrm{Te}_{4}$ obtained by high-resolution ARPES experiments. Figure 2(a) illustrates a typical ARPES $k$ - $E$ cut through the surface zone center $\bar{\Gamma}$ we obtained with high-resolution laser ARPES [37] below $T_{N}(h \nu=6.3 \mathrm{eV}, T=10 \mathrm{~K})$. Even at the first glance, one notices that there undoubtedly exists a gapless state between the electronlike and holelike conduction and valence bands, whose dispersion is even more linear than conventional TIs like $\mathrm{Bi}_{2} \mathrm{Se}_{3}$ and $\mathrm{Bi}_{2} \mathrm{Te}_{3}$. The two branches of this state intersect at $\bar{\Gamma}$ at a binding energy of $0.290 \mathrm{eV}$, forming a prototypical Dirac cone at $\bar{\Gamma}$ without any trace of gap. This band is one of the sharpest electronic states ever seen in topological materials, with a full width half maximum (FWHM) of $0.010 \AA^{-1}$ (detailed in Ref. [38]). Note that an ungapped Dirac cone is also reported in Ref. [22] for few-SL films of $\mathrm{MnBi}_{2} \mathrm{Te}_{4}$, yet the measurements there were done only at the PM state above $T_{N}$. The main purpose of the present paper is to study the spectroscopic properties of this state and to propose, based on reasonable symmetry arguments and DFT calculations, the origin of this state.

Having established the existence of the gapless Dirac state, an important question is whether this gapless band remains unchanged for all $k_{z}$ 's in the Brillouin zone (i.e., it is a 2D state), or if it gradually opens a gap as $k_{z}$ varies (i.e., it is a 3D state). We prove that this state is in fact a quasi-2D state with little $k_{z}$ dispersion by performing a detailed photon energy dependent ARPES map from 6 to $20 \mathrm{eV}$, corresponding to $3.7<k_{z}<5.8$ (in unit of $2 \pi / c$ ), covering more than two Brillouin zones in the $k_{z}$ direction [Fig. 2(b)]. Note that the lattice constant $c$ here represents the height of a single SL of $\mathrm{MnBi}_{2} \mathrm{Te}_{4}(c=1.37 \mathrm{~nm})$, which is about $1 / 3$ the height of the conventional unit cell. For all $k_{z}$ values within this range where the Dirac state is resolvable, we see negligible change in the $(k, E)$ position of the Dirac band [38]. Therefore, the Dirac state behaves as a quasi-2D, surfacelike electronic state. On the other hand, the bands that form a gap at $\bar{\Gamma}$, especially the one below the Dirac band, show clear periodic dispersion along $k_{z}$ [Fig. 2(b)]. As a result, the size of the $\bar{\Gamma}$ gap varies between $0.13 \mathrm{eV}$ at the bulk $\Gamma$ and $0.20 \mathrm{eV}$ at $Z$ [Figs. 2(c)-2(e)]. In light of this behavior, we assign this gapped band to be a bulklike electronic state. 

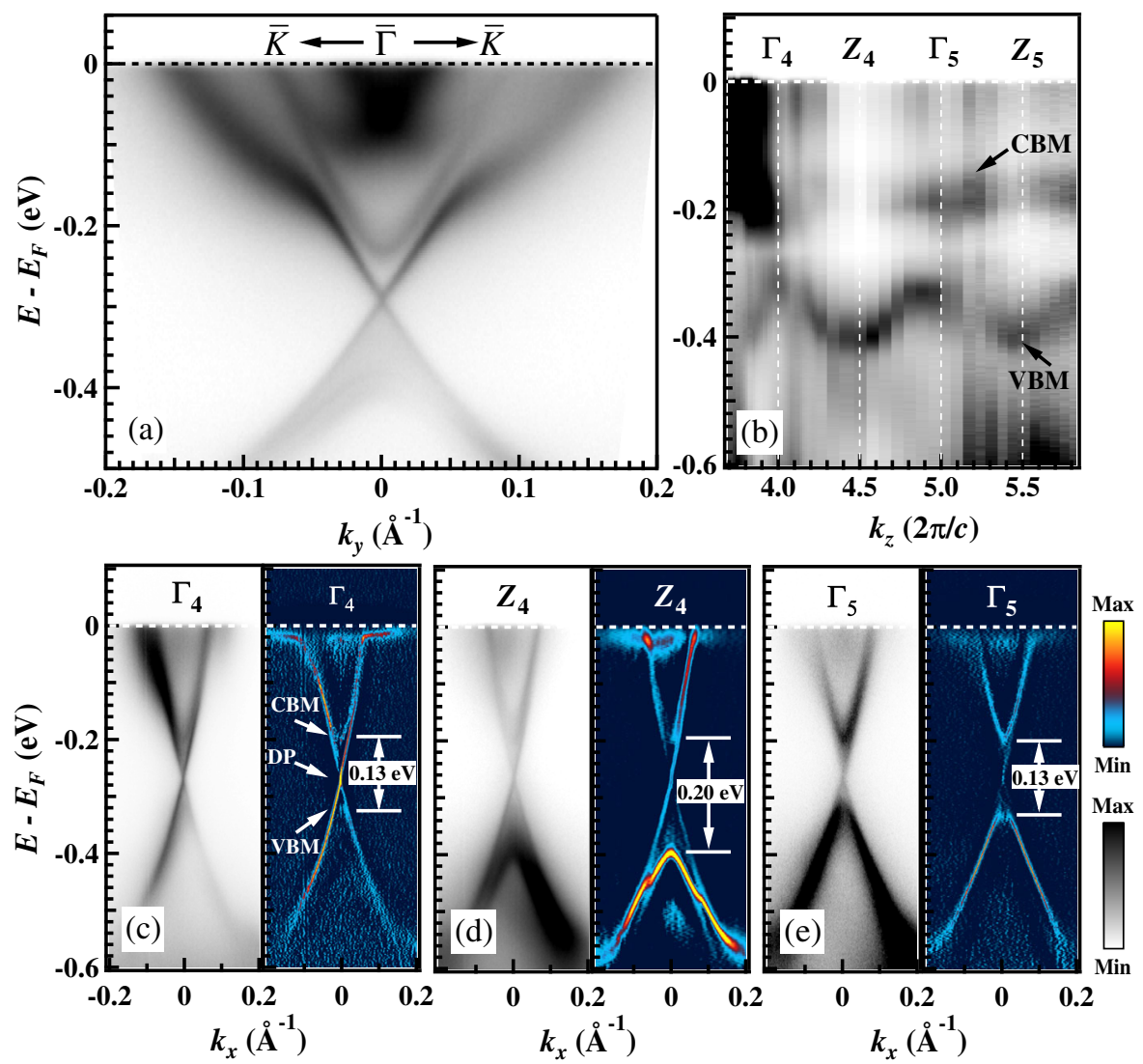

FIG. 2. Surface and bulk electronic structure of $\mathrm{MnBi}_{2} \mathrm{Te}_{4}$. (a) A typical ARPES $k-E$ map along $\bar{K}-\bar{\Gamma}-\bar{K}\left(k_{y}\right)$, taken at $10 \mathrm{~K}$ under photon energy $h \nu=6.3 \mathrm{eV}$. A linear, X-shaped, gapless state exists between the valence and the conduction bands. The Dirac point (DP) energy locates at $E_{D}=288 \mathrm{meV}$ for this sample. (b) $k_{z}$ dispersion map at $\bar{\Gamma}$, taken with 6-20 eV photons. VBM, valence band maxima; CBM, conduction band minima. Periodic dispersion pattern on the VBM is seen clearly. The bulk $\Gamma$ and $Z$ points are determined by assigning an inner potential $V_{0}=9 \mathrm{eV}$, estimated from the total bandwidth of the valence bands. (c)-(e) $k$ - $E$ maps along $\bar{M}-\bar{\Gamma}-\bar{M}\left(k_{x}\right)$ taken at the $\Gamma_{4}, Z_{4}$, and $\Gamma_{5}$ points marked in (b) (corresponding to $h \nu=7.5,10.5$, and $13.5 \mathrm{eV}$, respectively). It is clear that the gapless state forming the Dirac cone has no $k_{z}$ dispersion, while the VBM evolves from $-0.33 \mathrm{eV}(\Gamma)$ to $-0.4 \mathrm{eV}(Z)$, consequently changing the bulk gap from 0.13 to $0.20 \mathrm{eV}$.

(a) Pristine, 10 K

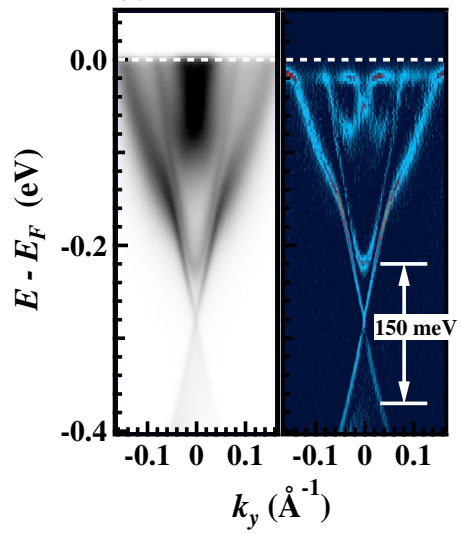

(b) Pristine, $300 \mathrm{~K}$

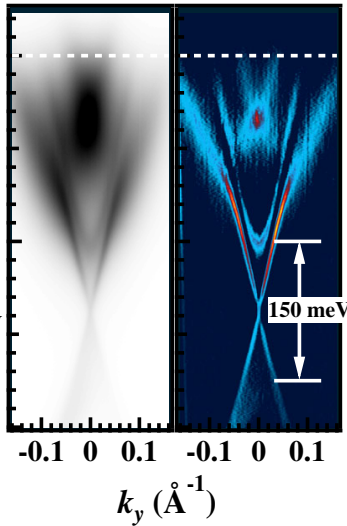

(c) Air cleaved, $10 \mathrm{~K}$

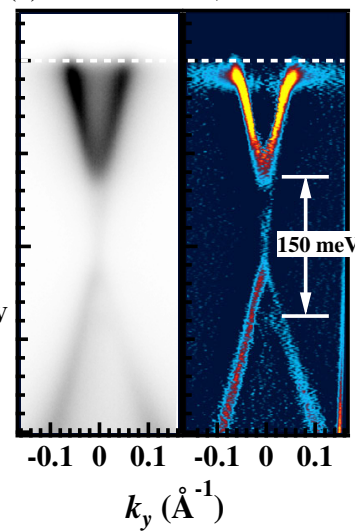

FIG. 3. Robustness of the Dirac surface state. ARPES raw (left) and second derivative (right) $k$ - $E$ maps taken with 6.3 eV laser light along $\bar{K}-\bar{\Gamma}-\bar{K}\left(k_{y}\right)$ for (a) a pristine sample cleaved and measured at $10 \mathrm{~K}$ (below $T_{N}$ ), (b) a pristine sample cleaved and measured at $300 \mathrm{~K}$ (well above $T_{N}$ ), and (c) a sample cleaved in air at room temperature, and measured at $10 \mathrm{~K}$. Despite the overall carrier doping induced by different cleaving conditions, the gapless Dirac cone is clearly seen for all cases, along with an unchanged bulk band gap sized $150 \mathrm{meV}$. 
Next we study whether this Dirac state remains across the magnetic transition temperature, and whether it is robust against considerable surface perturbation. Figure 3 gives positive answers to both questions. When an as-grown, pristine $\mathrm{MnBi}_{2} \mathrm{Te}_{4}$ crystal is cleaved in situ at $10 \mathrm{~K}$ (below $T_{N}$ ), it shows a clear ungapped Dirac cone whose Dirac point lies at $E_{b}=0.28 \mathrm{eV}$ [Fig. 3(a)] [50]. In case of $6.3 \mathrm{eV}$ photons, the bulk gap measures to be $150 \mathrm{meV}$. When we raise the temperature to $300 \mathrm{~K}$ [Fig. 3(b)], the Dirac point energy changes to $E_{b}=0.27 \mathrm{eV}$, yet the gapless nature of the Dirac band, as well as the size of the bulk gap, remains. Therefore, being in the AFM ground state or the hightemperature PM state does not seem to affect the integrity of the cone. To further test the robustness of the cone, we cleave a sample in air at room temperature before loading it into the vacuum chamber, and measure the band structure of the disturbed surface at $10 \mathrm{~K}$ [Fig. 3(c)]. Although the band structure becomes significantly $p$ doped compared with the pristine one, the Dirac surface state is still visible, without any sign of gap opening. The bulk gap also keeps its size of $150 \mathrm{meV}$. The message here is that this Dirac state is as robust as those in prototypical nonmagnetic TIs like $\mathrm{Bi}_{2} \mathrm{Se}_{3}$ [51]. Therefore, it is highly likely that this state is topologically protected by a symmetry of the crystal.

\section{PROPOSED GAPLESS DIRAC CONE FROM DIFFERENT SURFACE MAGNETIC STRUCTURES}

Next we discuss the possible physical origin of the gapless (0001) surface Dirac cone in $\mathrm{MnBi}_{2} \mathrm{Te}_{4}$ from the perspective of surface spin reorientation. For 3D magneticdoped TIs, it is reported that the helical surface electrons can induce a FM order at the surface through RudermanKittel-Kasuya-Yosida interaction even when the bulk is not magnetically ordered [52-55]. In $\mathrm{MnBi}_{2} \mathrm{Te}_{4}$, previous neutron diffraction measurements confirmed an A-type AFM spin configuration with the magnetization along the $c$ axis [24], which supports a massive surface Dirac cone if the bulk magnetic configuration remains at the surface [Fig. 4(a)]. Therefore, our results suggest that the surface-mediated spin configuration at the few top layers differs from that in the bulk state, hosting topologyprotected gapless surface states which can be detected by our surface-sensitive ARPES technique. In the following we consider several possible magnetic states of the surface layers that can support the linear-dispersed, gapless surface Dirac cone, and then discuss their chance of happening based on the current experimental observations and our corresponding total energy calculations. They are summarized in Figs. 4(b)-4(d) and Table I.

Before proposing the possibilities, we make a basic assumption that the gapless surface states are protected by $\mathcal{T}$, crystalline symmetry, or their combinations. Starting from nonmagnetic $\mathrm{MnBi}_{2} \mathrm{Te}_{4}$ having the same space group of $\mathrm{Bi}_{2} \mathrm{Te}_{3}(R \overline{3} \mathrm{~m})$, there are in total five types of symmetry operations, i.e., threefold rotation along the $z$ axis $\mathcal{C}_{3 z}$,

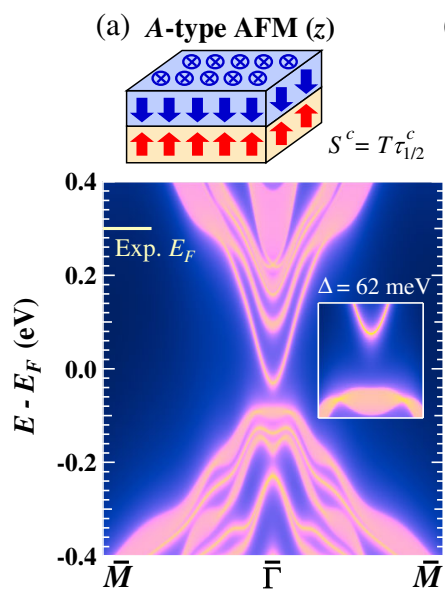

(b) Disordered moment

(c) $A$-type $\operatorname{AFM}(x)$
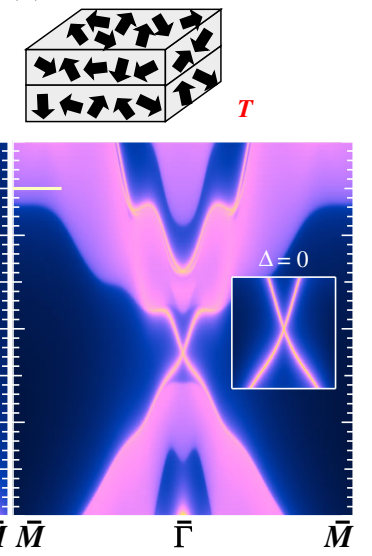

(d) $G$-type AFM

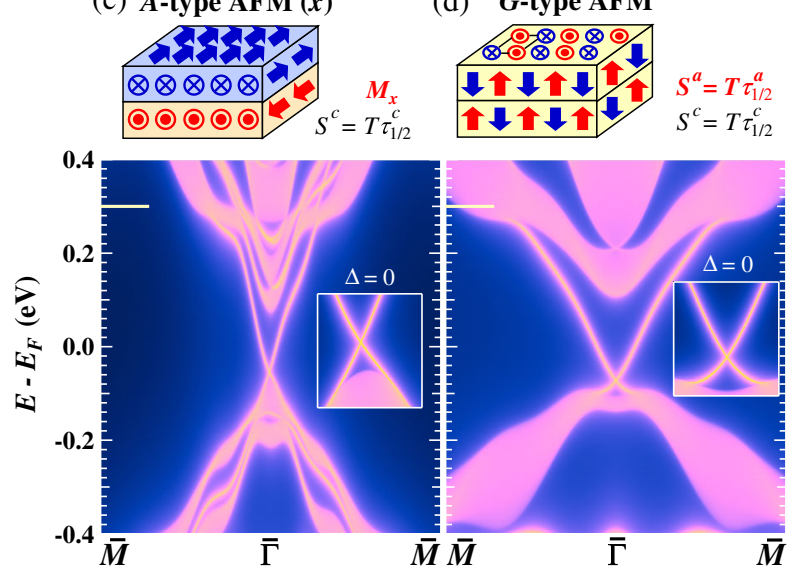

FIG. 4. DFT-calculated surface states of $\mathrm{MnBi}_{2} \mathrm{Te}_{4}$ for four prototypical magnetic configurations. (a) A-type AFM with the magnetic moments along the $z$ axis, (b) disordered magnetic moments, (c) A-type AFM with the magnetic moments along the $x$ axis, and (d) $G$-type AFM. The top drawing in each panel specifies the magnetic configuration and the symmetries that preserve in bulk (black) and (0001) surface (red). $\mathcal{T}$, time reversal; $\tau_{1 / 2}^{i}(i=a, b, c)$, half-cell translation along $i ; \mathcal{M}$, mirror plane.

twofold rotation along the $x$ axis $\mathcal{C}_{2 x}$, inversion $\mathcal{I}$, rotoinversion $\mathcal{S}_{6 z}=\mathcal{C}_{3 z} \mathcal{I}$, and mirror symmetry $\mathcal{M}_{x}=\mathcal{C}_{2 x} \mathcal{I}$. At the (0001) surface of $\mathrm{MnBi}_{2} \mathrm{Te}_{4}$, since $\mathcal{C}_{2}, \mathcal{I}$, and $\mathcal{S}_{6}$ symmetries are broken, the point group is reduced to $\mathcal{C}_{3 v}$. Therefore, when we take the local magnetic moment of $\mathrm{Mn}$ atoms into account, the symmetry preserved at this surface can only be $\mathcal{C}_{3 z}, \mathcal{M}_{x}$, in-plane translation symmetry $\tau_{a b}, \mathcal{T}$ and their combinations, i.e., $\mathcal{C}_{3 z} \mathcal{T}, \mathcal{M}_{x} \mathcal{T}$, and $\tau_{a b} \mathcal{T}$. We thus expect that the observed gapless surface Dirac cone is protected by one of these symmetries. Such a symmetry $\mathcal{G}$ should fulfill $\mathcal{G}^{2}=-1$ in our spin- $1 / 2$ systems for at least certain high-symmetry points in the $k$ space, resulting in Kramers degeneracy and nontrivial topological classification. While high-order topology and gapless hinge or edge states can be protected by symmetry operations like threefold axis [56], $\mathcal{M}_{x} \mathcal{T}, \mathcal{C}_{3 z}$, and $\mathcal{C}_{3 z} \mathcal{T}$ are excluded in our following analysis because they do not support gapless 
TABLE I. Properties of $\mathrm{MnBi}_{2} \mathrm{Te}_{4}$ with different magnetic configurations considered in our work, including the calculated total energy with respect to the magnetic structure of ground state, i.e., $A$-type AFM (z) configuration, the gap size of surface states $\left(E_{g}\right)$, the topological classification, and the corresponding symmetry. The number in parentheses is the square of the symmetry operation. NM, nonmagnetic; MCN, mirror Chern number.

\begin{tabular}{lcccc}
\hline \hline Phase & Energy $(\mathrm{meV} / \mathrm{Mn})$ & Surface $E_{g}(\mathrm{meV})$ & Topological classification & Symmetry \\
\hline$A$-type AFM $(z)$ & 0.00 & 62 & $\mathbb{Z}_{2}$ & $\mathcal{S}^{c}\left(-e^{\left.-2 i k \tau_{1 / 2}^{c}\right)}\right.$ \\
$A$-type AFM $(y)$ & 0.41 & 0.3 & $\ldots$ & $\ldots$ \\
$A$-type AFM $(x)$ & 0.41 & 0 & $\mathrm{MCN}$ & $\mathcal{M}_{x}(-1)$ \\
$G$-type AFM & 8.34 & 0 & $\mathbb{Z}_{2}$ & $\mathcal{S}^{a}\left(-e^{\left.-2 i k \tau_{1 / 2}^{a}\right)}\right.$ \\
$C$-type AFM & 8.38 & 0 & $\mathbb{Z}_{2}$ & $\mathcal{S}^{a}\left(-e^{\left.-2 i k \tau_{1 / 2}^{a}\right)}\right.$ \\
PM & 5.73 & 0 & $\mathbb{Z}_{2}$ & $\mathcal{T}(-1)$ \\
NM & $4.12 \times 10^{3}$ & 0 & $\mathbb{Z}_{2}$ & $\mathcal{T}(-1)$ \\
\hline \hline
\end{tabular}

surface states [57]. Hence, the remaining symmetry operations are $\mathcal{T}, \mathcal{M}_{x}$, and $\tau_{a b} \mathcal{T}$.

The first possibility is that the ordered spin in the bulk state may show fragility at the surface layers, leading to a lower transition temperature and a PM state with spin disorder. In this case, the electron hopping and magnetic moment decouple with each other and $\mathcal{T}$ symmetry is restored, leading to a gapless surface Dirac cone from a 3D $\mathcal{T}$-preserved TI. To obtain the total energy of such a PM state, we use a $4 \times 4$ supercell with special quasirandom structures to simulate the spin disorder effect [58]. Such an approach provides the best choice of randomness for a finitesize supercell in guaranteeing the best match of correlation functions of the infinite alloy, and thus can be applied in both cases of composition disorder and spin disorder. The total energy of such a magnetic configuration is found to be $5.7 \mathrm{meV} / \mathrm{Mn}$ compared with the ground state.

Without ordered magnetization, the model Hamiltonian for a single surface of $\mathrm{MnBi}_{2} \mathrm{Te}_{4}$ can be written up to the third order as $H=H_{1}+H_{3}$. The first term $H_{1}$ is the wellknown surface Rashba term,

$$
H_{1}=\alpha_{1}\left(k_{x} \sigma_{y}-k_{y} \sigma_{x}\right)
$$

where $\sigma$ is the Pauli matrix for spin degree of freedom. $\mathrm{H}_{3}$ is the cubic- $k$ term written as

$$
\begin{aligned}
H_{3}= & \alpha_{2}\left[\left(k_{x}^{2}+k_{y}^{2}\right) k_{x} \sigma_{y}-\left(k_{y}^{2}+k_{x}^{2}\right) k_{y} \sigma_{x}\right] \\
& +\alpha_{3}\left(k_{x}^{2}-3 k_{y}^{2}\right) k_{x} \sigma_{z}
\end{aligned}
$$

which is derived from the $k \cdot p$ perturbation by considering all the possible $k$-polynomial terms that respect the crystal symmetry [59]. The basis used for this $k \cdot p$ model is the $m_{j}= \pm 1 / 2$ states belonging to the representation $E_{1 / 2}$ [60], because the bulk bands near the Fermi level are mainly contributed by Te- $p_{z}$ and Bi- $p_{z}$ orbitals with $l=1$ and $m_{l}=0$. The Hamiltonian $H$ apparently supports a gapless Dirac cone at the $\bar{\Gamma}$ point. Figure 4(b) shows the gapless surface Dirac cone of nonmagnetic $\mathrm{MnBi}_{2} \mathrm{Te}_{4}$ calculated by DFT as an approximation of the PM state, indicating a $3 \mathrm{D} \mathcal{T}$-preserved TI. A careful comparison between ARPES data and DFT calculation reveals good consistency for the spin disorder scenario [38], which also explains the observed almost unchanged band structure with the temperature across the transition point of $24 \mathrm{~K}$, and the robustness of the surface Dirac cone against severe degradation.

The second possible type of symmetry that can protect the gapless surface state is the mirror symmetry. In this case, we begin with $A$-type AFM with in-plane magnetic moment that is perpendicular to one mirror plane $\mathcal{M}_{x}$ [because of $\mathcal{C}_{3 z}$, there are three equivalent mirror planes at the (0001) surface]. Such a configuration needs to overcome only a small magnetic anisotropy energy compared with the magnetic ground state, i.e., $0.4 \mathrm{meV} / \mathrm{Mn}$. In this case, $\mathrm{MnBi}_{2} \mathrm{Te}_{4}$ is calculated to be an AFM TI and an $\mathcal{M}_{x}$-protected AFM topological crystalline insulator (TCI) with nontrivial mirror Chern number $(\mathrm{MCN})$-in other words, a dual AFM TI. This is analogous to $\mathrm{Bi}_{2} \mathrm{Se}_{3}$ as a nonmagnetic dual TI [61]. As a result, the TCI phase gives rise to a gapless Dirac cone slightly off the $\bar{\Gamma}$ point, as shown in Fig. 4(c). In $\mathrm{MnBi}_{2} \mathrm{Te}_{4}$ the shift is $0.005 \AA^{-1}$ along the $k_{y}$ direction that is perpendicular to the magnetic moment. Note that if this is realized at the surface, and the sizes of the magnetic domains having opposite magnetic moments are smaller than the size of the ARPES incident beam ( $\sim 10 \mu \mathrm{m}$ for laser ARPES), we would expect a doubling of the Dirac cone surface states with momentum separation $0.01 \AA^{-1}$, which is marginally detectable under the momentum resolution of our ARPES data. The fact that this is not observed in our dataset would indicate either the absence of in-plane $A$-type AFM at the surface or magnetic domains that are significantly larger than $\sim 10 \mu \mathrm{m}$.

We next consider the magnetic moment along a more general in-plane direction. Without the protection of mirror 
symmetry, we can expect a gap opening at the (0001) surface. This can be understood by adding a magnetizationinduced Zeeman term $H_{\text {mag }}=g \mathbf{B} \cdot \boldsymbol{\sigma}$ with in-plane magnetic field to the nonmagnetic Hamiltonian $H$ [see Eqs. (1) (2)]. If $H_{3}$ is omitted, $H_{1}+H_{\text {mag }}$ will lead to the shift of the Dirac point perpendicular to the direction of the magnetic moment without a gap opening. On the other hand, adding $H_{\text {mag }}$ to $H=H_{1}+H_{3}$ will open a gap at the Dirac cone because the inclusion of the high-order term $\mathrm{H}_{3}$ introduces a hexagonal warping term $\sigma_{z}$ that tilts the spin out of the plane [59,61]. Only if $\mathbf{B}$ is perpendicular to one of the mirror planes $\left(k_{x}=0\right.$ or $\left.k_{x}= \pm \sqrt{3} k_{y}\right)$, the $\sigma_{z}$ term vanishes at the shifted Dirac point and the gapless Dirac cone retains. As shown in Table I, the features of the surface band gaps with different in-plane magnetic moment derived from the model Hamiltonian are consistent with our DFT calculation. However, our DFT calculation reveals that the size of the gap induced by the high-order term $\mathrm{H}_{3}$ is as small as $\sim 0.3 \mathrm{meV}$, which is negligible within the resolution of ARPES. Hence, a general A-type AFM configuration with in-plane magnetic moment is also compatible with our experimental observation. Since the total energies for A-type AFM with different in-plane spin orientations are almost the same, we suggest that the surface layers of real samples probably consist of magnetic domains with different in-plane FM spin moments.

The third type of symmetry is the combination of $\mathcal{T}$ and translation symmetry $\tau_{a b}$. This type of symmetry requires intralayer AFM spin configuration, exemplified by the $G$-type AFM where both intralayer and interlayer alignment of magnetic moments are AFM, as shown in Fig. 4(d). In such a magnetic configuration there exist $\mathcal{S}^{i}=\mathcal{T} \tau_{1 / 2}^{i}$ symmetries along all the three lattice vectors $i=a, b, c$ that correspond to two independent elements $\mathcal{S}^{a}$ and $\mathcal{S}^{c}$ in the magnetic space group ( $\mathcal{S}^{a}$ and $\mathcal{S}^{b}$ are equivalent elements). The square of $S^{i}$ equals to -1 at certain time-reversal invariant momenta that meet the requirement $k \tau_{1 / 2}^{i}=n \pi$, leading to two $\mathbb{Z}_{2}$ invariants in this system. Since the only band inversion occurs at the $\Gamma$ point in $G$-type AFM $\mathrm{MnBi}_{2} \mathrm{Te}_{4}$, all three topological invariants are nontrivial, giving rise to a gapless Dirac cone at the (0001) surface because $\mathcal{S}^{a}$ remains invariant at this surface. Similarly, some other magnetic configurations with in-plane AFM alignment, such as $C$-type AFM, also host a gapless (0001) surface state protected by $\mathcal{S}^{a}$ symmetry with in-plane fractional translation. However, our DFT calculations show that forming intralayer AFM alignment in the surface layers needs to overcome the favored intralayer FM exchange coupling in the bulk, leading to a large energy cost of about $8.3 \mathrm{meV} / \mathrm{Mn}$ [38]. Furthermore, if the intralayer AFM is realized at the surface, the in-plane Fermi surface should exhibit a band folding effect, which is not observed by our ARPES measurements. Overall, to obtain the direct evidence for addressing the origin of the gapless feature, it is crucial to involve surface-sensitive detection, such as x-ray magnetic circular dichroism and the NV center technique, to determine the surface magnetic structure of such intrinsic magnetic TI in the future.

\section{DISCUSSION AND CONCLUSION}

Apart from the possible origins of the gapless surface Dirac cone due to surface spin reorientation in ideal $\mathrm{MnBi}_{2} \mathrm{Te}_{4}$ single crystals, we briefly discuss the possibility of structural deformation owing to the sample imperfection that could also hint at the deviation of the electronic structure between ARPES measurements and the theoretical calculations based on A-type AFM magnetic configuration. For example, the $\mathrm{MnBi}_{2} \mathrm{Te}_{4}$ thin film could be

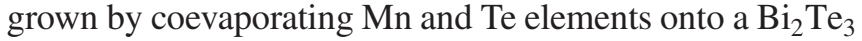
surface, corresponding to the coverage of a MnTe layer [21]. Although the $\mathrm{Mn}^{2+}$ ion is energetically favorable to intercalate into the central layer of a $\mathrm{MnBi}_{2} \mathrm{Te}_{4} \mathrm{SL}$, the growing condition determines the existence of cation mixing between $\mathrm{Mn}$ and $\mathrm{Bi}$, or antisite defects [20]. Such an occasion would lead to disordered Mn distribution when approaching the surface layers as well as magnetic disorder. In addition, it is reported that the synthetic $\mathrm{MnBi}_{2} \mathrm{Te}_{4}$ tends to experience a decomposition into $\mathrm{Bi}_{2} \mathrm{Te}_{3}$ and $\mathrm{MnTe}_{2}$ phases at a higher temperature [35]. Taking into account the surface potential, the surface $\mathrm{MnBi}_{2} \mathrm{Te}_{4}$ layer might also suffer structural reconstruction or dislocation, such as decaying to $\mathrm{Bi}_{2} \mathrm{Te}_{3}$ layer, rendering different band dispersions compared with an ideal $\mathrm{MnBi}_{2} \mathrm{Te}_{4}$ surface. Finally, the imperfect crystal could lead to a number of ferromagnetic domains of which the moments point to different directions, contributing different values of the Zeeman term $H_{\text {mag }}=g \mathbf{B} \cdot \boldsymbol{\sigma}$. As a result, the measurable topological surface states could be compensated by the synergetic effect of different domains.

In summary, we demonstrate unambiguously by systematic ARPES measurements that $\mathrm{MnBi}_{2} \mathrm{Te}_{4}$ is not an ideal AFM TI. A gapless surface Dirac cone exists experimentally in single crystal $\mathrm{MnBi}_{2} \mathrm{Te}_{4}$, hosting massless Dirac quasiparticles. The Dirac cone is found to be quasi-2D and stable under massive surface absorbents; the bulk and surface electronic structures show no observable change across the bulk magnetic transition temperature. Our first principles calculation further identifies several magnetic configurations that could yield such a gapless topological state. It is important to note that such reconstruction might not be limited only in $\mathrm{MnBi}_{2} \mathrm{Te}_{4}$, but in a group of other intrinsic magnetic topological materials, because several competing phases with different structural or magnetic orders could have small energy differences and thus are sensitive to surrounding environments and the presence of surface termination. Therefore, our research also sheds light on other magnetic topological materials, revealing that nature deals with AFM TIs in a more intricate way than previously thought. Motivated by our results, the theme of 
future works should be finding a way to overcome such surface reconstruction in favor of the long-sought axion insulators, or make use of such reconstruction to build devices with novel transport phenomena. Taken collectively, our discovery of the unexpected massless Dirac quasiparticles in an AFM TI indicates a space-varying magnetic structure more complex than previously assigned in these materials, brings about a more complete description of magnetic topological systems, and paves the way for the exploration of the interplay of massive and massless Dirac particles in a single material platform.

\section{ACKNOWLEDGMENTS}

We thank Jieming Sheng, Yue Zhao, Haizhou Lu, Jianpeng Liu, Weiqiang Chen, and Dapeng Yu for helpful discussions. ARPES experiments were performed with the approval of the Hiroshima Synchrotron Radiation Center (HSRC), Hiroshima, Japan under Proposals No. 19AG014 and No. 19AG004. Work at SUSTech was supported by the National Natural Science Foundation of China (NSFC) (No. 11504159, No. 11674149, and No. 11874195), NSFC Guangdong (No. 2016A030313650), the Guangdong Innovative and Entrepreneurial Research Team Program (No. 2016ZT06D348 and No. 2017ZT07C062), the Guangdong Provincial Key Laboratory of Computational Science and Material Design (Grant No. 2019B030301001), the Shenzhen Key Laboratory (Grant No. ZDSYS20170303165926217), and the Technology and Innovation Commission of Shenzhen Municipality (Grants No. JCYJ20150630145302240 and No. KYTDPT 20181011104202253). Work at UCLA was supported by the U.S. Department of Energy (DOE), Office of Science, Office of Basic Energy Sciences under Award No. DE-SC0011978.

Y.-J. H., P. L., and Y. F. contributed equally to this work.

Note added.-Recently, we became aware of similar studies [62-64] reporting gapless Dirac topological surface states in $\mathrm{MnBi}_{2} \mathrm{Te}_{4}$.

[1] Y. L. Chen et al., Massive Dirac Fermion on the Surface of a Magnetically Doped Topological Insulator, Science 329, 659 (2010).

[2] S.-Y. Xu et al., Hedgehog Spin Texture and Berry's Phase Tuning in a Magnetic Topological Insulator, Nat. Phys. 8, 616 (2012).

[3] Y. Tokura, K. Yasuda, and A. Tsukazaki, Magnetic Topological Insulators, Nat. Rev. Phys. 1, 126 (2019).

[4] N. P. Armitage and L. Wu, On the Matter of Topological Insulators as Magnetoelectrics, SciPost Phys. 6, 046 (2019).

[5] N. Regnault and B.A. Bernevig, Fractional Chern Insulator, Phys. Rev. X 1, 021014 (2011).
[6] X.-L. Qi, T. L. Hughes, and S.-C. Zhang, Topological Field Theory of Time-Reversal Invariant Insulators, Phys. Rev. B 78, 195424 (2008); Erratum, Phys. Rev. B 81, 159901(E) (2010).

[7] R. Yu, W. Zhang, H.-J. Zhang, S.-C. Zhang, X. Dai, and Z. Fang, Quantized Anomalous Hall Effect in Magnetic Topological Insulators, Science 329, 61 (2010).

[8] C. Fang, M. J. Gilbert, and B. A. Bernevig, Bulk Topological Invariants in Noninteracting Point Group Symmetric Insulators, Phys. Rev. B 86, 115112 (2012).

[9] C.-Z. Chang et al., Experimental Observation of the Quantum Anomalous Hall Effect in a Magnetic Topological Insulator, Science 340, 167 (2013).

[10] D. Xiao et al., Realization of the Axion Insulator State in Quantum Anomalous Hall Sandwich Heterostructures, Phys. Rev. Lett. 120, 056801 (2018).

[11] J. G. Checkelsky, R. Yoshimi, A. Tsukazaki, K. S. Takahashi, Y. Kozuka, J. Falson, M. Kawasaki, and Y. Tokura, Trajectory of the Anomalous Hall Effect towards the Quantized State in a Ferromagnetic Topological Insulator, Nat. Phys. 10, 731 (2014).

[12] X. Kou et al., Scale-Invariant Quantum Anomalous Hall Effect in Magnetic Topological Insulators beyond the Two-Dimensional Limit, Phys. Rev. Lett. 113, 137201 (2014);Erratum 113, 199901 (2014).

[13] C.-Z. Chang, W. Zhao, D. Y. Kim, H. Zhang, B. A. Assaf, D. Heiman, S.-C. Zhang, C. Liu, M. H. W. Chan, and J. S. Moodera, High-Precision Realization of Robust Quantum Anomalous Hall State in a Hard Ferromagnetic Topological Insulator, Nat. Mater. 14, 473 (2015).

[14] R. S. K. Mong, A. M. Essin, and J. E. Moore, Antiferromagnetic Topological Insulators, Phys. Rev. B 81, 245209 (2010).

[15] B. Huang et al., Layer-Dependent Ferromagnetism in a van der Waals Crystal Down to the Monolayer Limit, Nature (London) 546, 270 (2017).

[16] Z.S. Aliev et al., Novel Ternary Layered Manganese Bismuth Tellurides of the $\mathrm{MnTe}-\mathrm{Bi}_{2} \mathrm{Te}_{3}$ System: Synthesis and Crystal Structure, J. Alloys Compd. 789, 443 (2019).

[17] H. Sun, B. Xia, Z. Chen, Y. Zhang, P. Liu, Q. Yao, H. Tang, Y. Zhao, H. Xu, and Q. Liu, Rational Design Principles of Quantum Anomalous Hall Effect from Superlattice-like Magnetic Topological Insulators, Phys. Rev. Lett. 123, 096401 (2019).

[18] S. H. Lee et al., Spin Scattering and Noncollinear Spin Structure-Induced Intrinsic Anomalous Hall Effect in Antiferromagnetic Topological Insulator $\mathrm{MnBi}_{2} \mathrm{Te}_{4}$, Phys. Rev. Res. 1, 012011 (2019).

[19] M. M. Otrokov et al., Prediction and Observation of the First Antiferromagnetic Topological Insulator, arXiv:1809 .07389 .

[20] A. Zeugner et al., Chemical Aspects of the Candidate Antiferromagnetic Topological Insulator $\mathrm{MnBi}_{2} \mathrm{Te}_{4}$, Chem. Mater. 31, 2795 (2019).

[21] Y. Gong et al., Experimental Realization of an Intrinsic Magnetic Topological Insulator, Chin. Phys. Lett. 36, 076801 (2019).

[22] J. Li, C. Wang, Z. Zhang, B. L. Gu, W. Duan, and Y. Xu, Magnetically Controllable Topological Quantum Phase 
Transitions in the Antiferromagnetic Topological Insulator $\mathrm{MnBi}_{2} \mathrm{Te}_{4}$, Phys. Rev. B 100, 121103(R) (2019).

[23] M. M. Otrokov, I. P. Rusinov, M. Blanco-Rey, M. Hoffmann, A. Yu. Vyazovskaya, S. V. Eremeev, A. Ernst, P. M. Echenique, A. Arnau, and E. V. Chulkov, Unique Thickness-Dependent Properties of the van der Waals Interlayer Antiferromagnet $\mathrm{MnBi}_{2} \mathrm{Te}_{4}$ Films, Phys. Rev. Lett. 122, 107202 (2019).

[24] J.-Q. Yan, Q. Zhang, T. Heitmann, Z. Huang, K. Y. Chen, J.-G. Cheng, W. Wu, D. Vaknin, B. C. Sales, and R. J. McQueeney, Crystal Growth and Magnetic Structure of $\mathrm{MnBi}_{2} \mathrm{Te}_{4}$, Phys. Rev. Mater. 3, 064202 (2019).

[25] Y.-J. Deng et al., Magnetic-Field-Induced Quantized Anomalous Hall Effect in Intrinsic Magnetic Topological Insulator $\mathrm{MnBi}_{2} \mathrm{Te}_{4}$, arXiv:1904.11468.

[26] C. Liu et al., Quantum Phase Transition from Axion Insulator to Chern Insulator in $\mathrm{MnBi}_{2} \mathrm{Te}_{4}$, arXiv:1905.00715.

[27] J. Ge et al., High-Chern-Number and High-Temperature Quantum Hall Effect without Landau Levels, arXiv: 1907.09947.

[28] R. C. Vidal, H. Bentmann, T. R. F. Peixoto, A. Zeugner, S. Moser, C. H. Min, S. Schatz, K. Kissner, M. Unzelmann, C. I. Fornari et al., Surface States and Rashba-Type Spin Polarization in Antiferromagnetic $\mathrm{MnBi}_{2} \mathrm{Te}_{4}$, Phys. Rev. B 100, 121104(R) (2019).

[29] J.-Q. Yan, S. Okamoto, M. A. McGuire, A. F. May, R. J. McQueeney, and B.C. Sales, Evolution of Structural, Magnetic and Transport Properties in $\mathrm{MnBi}_{2-x} \mathrm{Sb}_{x} \mathrm{Te}_{4}$, Phys. Rev. B 100, 104409 (2019).

[30] C.-W. Hu et al., A van der Waals Antiferromagnetic Topological Insulator with Weak Interlayer Magnetic Coupling, arXiv:1905.02154.

[31] B. Chen et al., Intrinsic Magnetic Topological Insulator Phases in the $\mathrm{Sb}$ Doped $\mathrm{MnBi}_{2} \mathrm{Te}_{4}$ Bulks and Thin Flakes, Nat. Commun. 10, 4469 (2019).

[32] J. Cui, M. Shi, H. Wang, F. Yu, T. Wu, X. Luo, J. Ying, and X. Chen, Transport Properties of Thin Flakes of the Antiferromagnetic Topological Insulator $\mathrm{MnBi}_{2} \mathrm{Te}_{4}$, Phys. Rev. B 99, 155125 (2019).

[33] D. Zhang, M. Shi, T. Zhu, D. Xing, H. Zhang, and J. Wang, Topological Axion States in Magnetic Insulator $\mathrm{MnBi}_{2} \mathrm{Te}_{4}$ with the Quantized Magnetoelectric Effect, Phys. Rev. Lett. 122, 206401 (2019).

[34] J.-H. Li, Y. Li, S. Du, Z. Wang, B.-L. Gu, S.-C. Zhang, K. He, W. Duan, and Y. Xu, Intrinsic Magnetic Topological Insulators in van der Waals Layered $\mathrm{MnBi}_{2} \mathrm{Te}_{4}$-Family Materials, Sci. Adv. 5, eaaw5685 (2019).

[35] D. S. Lee, T.-H. Kim, C.-H. Park, C.-Y. Chung, Y. S. Lim, W.-S. Seo, and H.-H. Park, Crystal Structure, Properties and Nanostructuring of a New Layered Chalcogenide Semiconductor, $\mathrm{Bi}_{2} \mathrm{MnTe}_{4}$, CrystEngComm 15, 5532 (2013).

[36] J.-Z. Wu et al., Natural van der Waals Heterostructures with Tunable Magnetic and Topological States, arXiv: 1905.02385 .

[37] H. Iwasawa, E. F. Schwier, M. Arita, A. Ino, H. Namatame, M. Taniguchi, Y. Aiura, and K. Shimada, Development of Laser-Based Scanning $\mu$-ARPES System with Ultimate Energy and Momentum Resolutions, Ultramicroscopy 182, 85 (2017).
[38] See Supplemental Material at http://link.aps.org/ supplemental/10.1103/PhysRevX.9.041038 for details, which includes Refs. [17-19,23,27,30,39-49].

[39] G. Kresse and D. Joubert, From Ultrasoft Pseudopotentials to the Projector Augmented-Wave Method, Phys. Rev. B 59, 1758 (1999).

[40] J. P. Perdew, K. Burke, and M. Ernzerhof, Generalized Gradient Approximation Made Simple, Phys. Rev. Lett. 77, 3865 (1996) Erratum 78, 1396 (1997).

[41] V. I. Anisimov, J. Zaanen, and O. K. Andersen, Band Theory and Mott Insulators: Hubbard U Instead of Stoner I., Phys. Rev. B 44, 943 (1991).

[42] S. L. Dudarev, G. A. Botton, S. Y. Savrasov, C. J. Humphreys, and A.P. Sutton, Electron-Energy-Loss Spectra and the Structural Stability of Nickel Oxide: An LSDA + U Study, Phys. Rev. B 57, 1505 (1998).

[43] G. Kresse and J. Furthmüller, Efficiency of Ab initio Total Energy Calculations for Metals and Semiconductors Using a Plane-Wave Basis Set, Comput. Mater. Sci. 6, 15 (1996).

[44] M. M. Otrokov et al., Highly-Ordered Wide Bandgap Materials for Quantized Anomalous Hall and Magnetoelectric Effects, 2D Mater. 4, 025082 (2017).

[45] A. A. Mostofi, J. R. Yates, G. Pizzi, Y.-S. Lee, I. Souza, D. Vanderbilt, and N. Marzari, An Updated Version of wannier90: A Tool for Obtaining Maximally-Localised Wannier Functions, Comput. Phys. Commun. 185, 2309 (2014).

[46] N. Marzari and D. Vanderbilt, Maximally Localized Generalized Wannier Functions for Composite Energy Bands, Phys. Rev. B 56, 12847 (1997).

[47] Q. Wu, S. Zhang, H.-F. Song, M. Troyer, and A. A. Soluyanov, WannierTools: An Open-Source Software Package for Novel Topological Materials, Phys. Chem. Comm. 224, 405 (2018).

[48] A. Takayama, T. Sato, S. Souma, T. Oguchi, and T. Takahashi, One-Dimensional Edge States with Giant Spin Splitting in a Bismuth Thin Film, Phys. Rev. Lett. 114, 066402 (2015).

[49] L. Miao et al., Quasiparticle Dynamics in Reshaped Helical Dirac Cone of Topological Insulators, Proc. Natl. Acad. Sci. U.S.A. 110, 2758 (2013).

[50] Note that the Dirac point binding energy is $\sim 290 \mathrm{meV}$ for the sample used in Fig. 2(a) (Ref. [38], Sec. C), 269 meV for that used in Figs. 2(b)-2(e) (Ref. [38], Sec. D), and $\sim 280 \mathrm{meV}$ for that used in Fig. 3(a). This difference is understandable as the carrier concentration is slightly different in each crystal.

[51] C. Chen et al., Robustness of Topological Order and Formation of Quantum Well States in Topological Insulators Exposed to Ambient Environment, Proc. Natl. Acad. Sci. U.S.A. 109, 3694 (2012).

[52] L. A. Wray, S.-Y. Xu, Y. Xia, D. Hsieh, A. V. Fedorov, Y. S. Hor, R. J. Cava, A. Bansil, H. Lin, and M. Z. Hasan, A Topological Insulator Surface under Strong Coulomb, Magnetic and Disorder Perturbations, Nat. Phys. 7, 32 (2011).

[53] Q. Liu, C.X. Liu, C. Xu, X. L. Qi, and S. C. Zhang, Magnetic Impurities on the Surface of a Topological Insulator, Phys. Rev. Lett. 102, 156603 (2009).

[54] J.-J. Zhu, D. X. Yao, S. C. Zhang, and K. Chang, Electrically Controllable Surface Magnetism on the Surface of 
Topological Insulators, Phys. Rev. Lett. 106, 097201 (2011).

[55] G. Rosenberg and M. Franz, Surface Magnetic Ordering in Topological Insulators with Bulk Magnetic Dopants, Phys. Rev. B 85, 195119 (2012).

[56] F. Schindler et al., Higher-Order Topology in Bismuth, Nat. Phys. 14, 918 (2018).

[57] R.-X. Zhang and C.-X. Liu, Topological Magnetic Crystalline Insulators and Corepresentation Theory., Phys. Rev. B 91, 115317 (2015).

[58] A. Zunger, S. H. Wei, L. G. Ferreira, and J. E. Bernard, Special Quasirandom Structures, Phys. Rev. Lett. 65, 353 (1990).

[59] L. Fu, Hexagonal Warping Effects in the Surface States of the Topological Insulator $\mathrm{Bi}_{2} \mathrm{Te}_{3}$, Phys. Rev. Lett. 103, 266801 (2009).
[60] S. L. Altmann and P. Herzig, Point-Group Theory Tables (Clarendon Press, Oxford, 1994).

[61] T. Rauch, M. Flieger, J. Henk, I. Mertig, and A. Ernst, Dual Topological Character of Chalcogenides: Theory for $\mathrm{Bi}_{2} \mathrm{Te}_{3}$, Phys. Rev. Lett. 112, 016802 (2014).

[62] Y. J. Chen et al., this issue, Topological Electronic Structure and Its Temperature Evolution in Antiferromagnetic Topological Insulator $\mathrm{MnBi}_{2} \mathrm{Te}_{4}$, Phys. Rev. X 9, 041040 (2019).

[63] H. Li et al., following paper, Dirac Surface States in Intrinsic Magnetic Topological Insulators $\mathrm{EuSn}_{2} \mathrm{As}_{2}$ and $\mathrm{MnBi}_{2} \mathrm{Te}_{4}$, Phys. Rev. X 9, 041039 (2019).

[64] P. Swatek, Y. Wu, L.-L. Wang, K. Lee, B. Schrunk, J. Yan, and A. Kaminski, Gapless Dirac Surface States in the Antiferromagnetic Topological Insulator $\mathrm{MnBi}_{2} \mathrm{Te}_{4}$, arXiv:1907.09596. 